\title{
La communication non-verbale des utilisateurs du téléphone mobile dans les lieux publics
}

\section{Francis Jauréguiberry}

\section{OpenEdition \\ Journals}

Édition électronique

URL : https://journals.openedition.org/communicationorganisation/2439

DOI : 10.4000/communicationorganisation.2439

ISSN : 1775-3546

\section{Éditeur}

Presses universitaires de Bordeaux

\section{Édition imprimée}

Date de publication : 1 novembre 2000

ISSN : 1168-5549

Référence électronique

Francis Jauréguiberry, «La communication non-verbale des utilisateurs du téléphone mobile dans les lieux publics », Communication et organisation [En ligne], 18 | 2000, mis en ligne le 27 mars 2012, consulté le 05 août 2021. URL : http://journals.openedition.org/communicationorganisation/2439 ; DOI : https://doi.org/10.4000/communicationorganisation.2439

Ce document a été généré automatiquement le 5 août 2021 .

(C) Presses universitaires de Bordeaux 


\title{
La communication non-verbale des utilisateurs du téléphone mobile dans les lieux publics
}

\author{
Francis Jauréguiberry
}

1 La propension observée chez les récents utilisateurs du mobile à téléphoner de façon spontanée quel que soit l'endroit où ils se trouvent relativise la notion même de lieu' ${ }^{1}$. Étant « ici » et «ailleurs » à la fois, au moins par l'écoute et la parole, les "branchés » ont en effet tendance à oublier la détermination spatiale des lieux pour goûter à un ersatz d'ubiquité. Or un lieu, pour peu qu'il soit public, est toujours aussi social. Ceci signifie en particulier qu'un ensemble d'attitudes, de comportements et de règles « d'être en public » y est associé. Et c'est précisément cette façon d'être en public que les branchés, en s'octroyant le pouvoir de se trouver «là sans y être vraiment ", questionnent. La nature des réponses apportées à ce questionnement par ceux qui partagent les mêmes lieux que les branchés au moment où ces derniers téléphonent relève au mieux d'un détachement amusé ou d'une neutralité désabusée, au pire d'une attitude offusquée ou d'une réaction hostile. En quatre ans de recherche sur les branchés, nous n'avons pas recueilli un seul témoignage ni observé une seule situation qui puisse nous permettre de rendre compte de réactions positives à l'utilisation des mobiles en public. Personne, semble-t-il, n'est encore intervenu ouvertement auprès d'un inconnu téléphonant dans un lieu public pour lui manifester sa joie ou tout au moins sa satisfaction d'assister à une telle pratique. L'inverse est par contre quotidiennement vérifiable : les protestations et attitudes d'énervement suscitées par la généralisation des appels téléphoniques en public se multiplient. Certes, un assentiment a sans doute moins besoin d'être exprimé qu'une opposition, mais il est d'un autre côté plus aisé et agréable d'extérioriser une approbation qu'un désaccord. On peut donc raisonnablement en inférer que l'usage du mobile en public suscite chez ceux qui en sont témoins plus de réticence que d'adhésion, plus de désagrément que de plaisir. 
2 Pourquoi de telles réactions négatives? Le branché ne produit pourtant pas une nuisance sonore supplémentaire. Sa voix ne porte en tout cas pas plus que si son interlocuteur était en face de lui ${ }^{2}$. L'espace qu'il occupe ne varie pas. Ses gestes ne sont pas scandaleux. Son attitude, qui est souvent faite de retrait et d'effacement, tendrait plutôt à la discrétion. Au demeurant, avec l'explosion des ventes de mobiles, téléphoner en public est devenu banal ${ }^{3}$ Alors pourquoi donc de telles réactions? L'hypothèse ici défendue est que les branchés délivrent un message non-verbal - et la plupart du temps non-volontaire - de déni aux témoins de leur « envol »" L'attitude des branchés signifie toujours une mise entre parenthèses de l'ici au profit de l'ailleurs. Bien sûr, cet « ailleurs » est ici abordé de façon essentiellement métaphorique : la seule comparaison qui vaut vraiment entre les deux « espaces» se situe au niveau relationnel, L'ailleurs médiatique du branché est toujours considéré par lui. au moment où il appelle, comme plus nécessaire, plus agréable ou plus rentable que son ici physique. Mais les effets de cette comparaison sont visuellement tout à fait perceptibles : les personnes partageant l'ici physique du branché se sentent niées ou mises entre parenthèses par lui.

Dans les quelques pages qui suivent, nous nous attacherons donc d'abord à cerner la nature de ce message, en particulier en montrant comment son efficacité (production d'une gêne alentour) est directement liée au fait que son contenu échappe en partie à ceux qui l'émettent. Nous déterminerons ensuite par quelles attitudes ce message est donné à percevoir, à partir de quels référents sémiotiques il est interprété par ceux qui le «lisent » et en fonction de quels critères discriminants les réponses s'ordonnent. Enfin, nous avancerons en conclusion deux remarques d'ordre heuristique quant au devenir de la civilité dans les lieux publics ${ }^{5}$.

\section{La règle du jeu de l'être en public}

Dans son ouvrage consacré au déclin de la culture publique, Richard Sennett définie la civilité comme « l'activité qui protège le moi des autres moi, et lui permet donc de jouir de la compagnie d'autrui. Le port du masque est l'essence même de la civilité. Les masques permettent la pure sociabilité, indépendamment des sentiments subjectifs de puissance, de gêne, etc., de ceux qui les portent. La civilité préserve l'autre du poids du moi $»^{6}$. Pour Sennett, la civilité consiste donc avant tout à respecter une distance à autrui, et le port de masques sociaux est son instrument.

5 Mais le fait de « ne pas peser sur les autres » ne signifie pas pour autant être indifférent envers eux. Cela implique au contraire un subtil mode de prise en compte de leur présence et d'attention à leur égard. Ainsi, relèvent Louis Quéré et Dietrich Brezger, "l'inattention civile est une forme de sociabilité, un mode d'organisation de la coprésence. Elle consiste à créer de la distance entre les personnes, à se rendre poliment étrangers les uns aux autres, à s'installer dans une extériorité des uns par rapport aux autres, à instaurer une a-propriété mutuelle $\aleph^{7}$. La civilité nécessite donc à la fois distance et attention. Suffisamment de distance pour que l'autonomie de chacun dans sa spécificité soit préservée, et autant d'attention pour que cette distance ne soit pas synonyme d'indifférence ou de déni.

6 Posant la question de savoir comment se fait cette démonstration de polite estrangement et quel est son support opérationnel. Quéré et Brezger pensent que «le cœur de cet accomplissement est une organisation de perspectives et une adoption d'attitudes » et soulignent que « la gestion du regard et les postures corporelles jouent 
un rôle essentiel $»^{8}$. Il faut évidemment y ajouter la grande importance de l'ouïe ${ }^{9}$. Il s'agit de montrer à autrui, par un type de regard (non-focalisé. furtif ou coup d'œil rapide) et d'écoute (flottante et non tendue), ainsi que par une gestuelle et un mode particulier d'occupation de l'espace, que l'on se fond dans la normalité comportementale d'être en public. Le vécu de cette étrangeté mutuelle attentionnée, fondatrice du caractère public de certains lieux, relève donc, in fine, de la production des apparences et de leur visibilité, autrement dit d'observabilités.

Nul mieux qu'Erving Goffman n'a décrit les attitudes que chacun se doit d'adopter en public s'il ne veut pas "perdre la face» ou faire perdre celle des personnes qui constituent son entourage ${ }^{10}$. Ces attitudes, que chacun peut spontanément vivre ou interpréter comme étant individuelles, donc relevant d'un plus ou moins grand " art » personnel d'être en public, renvoie en fait, nous explique Goffman. à tout un ensemble de règles éminemment sociales qu'il appelle des « relations syntaxiques $»^{11}$. L'individu est en effet libre de faire les "phrases » qu'il désire, mais est obligé, s'il veut être compris, d'employer un « vocabulaire » et une « syntaxe » compris par tous. Autrement dit. l'action substantielle d'un individu n'acquiert de signification que rapportée au référent social qui l'informe. C'est en employant la même métaphore linguistique que. quelques années auparavant, l'anthropologue Edward Sapir décrivait déjà l'enchaînement des gestes des individus engagés dans des communications interindividuelles. Cet enchaînement relève, écrivait-il. "d'un code secret et compliqué, écrit nulle part, connu de personne, (mais) entendu par tous " ${ }^{12}$. Le " code " premier régissant "l'être en public » de nos sociétés modernes est celui du polite estrangement, de l'attention distante ou de la distance potentiellement intentionnée. C'est parce que chacun fait attention à autrui tout en gardant son quant-à-soi que le quant-à-soi d'autrui est préservé dans une indifférence feinte. Si cette indifférence est troublée par un événement imprévu, le "code » se décline alors dans un ensemble d'attitudes destinées à rétablir une situation d'équilibre. Ce que Goffman résume en écrivant que "l'individu, dans un lieu public, paraît indifférent aux inconnus qui l'entourent. Mais, vient-il. entre autres choses, à sentir le besoin d'accomplir un rituel correctif, il est suffisamment orienté vers eux pour pouvoir les transformer en un public de sa représentation ${ }^{13}$

\section{Le dédoublement du branché}

8 Or, que donne à voir le branché à ceux qui assistent à son " envol médiatique "? Non pas une distance attentionnée mais une absence intentionnée. Le branché fait, en quelque sorte, fi de sa présence publique pour vivre son absence privée. La réciprocité des perspectives, pensée comme condition de partage d'un environnement commun, n'est dès lors plus possible ${ }^{14}$. Les préalables à l'échange des perspectives sont en effet inégalement partagés : la perspective du branché est sensitivement dédoublée (ici et ailleurs à la fois) tandis que celle du ou des témoins de son " envol» demeure unique (ici). Certes, ce dédoublement sensitif n'est qu'auditif. Mais on ne regarde pas pareil quand on écoute ailleurs. Nous avons en effet pu observer comment le regard du branché pouvait parfois "accompagner " l'ouie, comme si, par couplage sensitif, le regard en venait lui aussi à se dédoubler. C'est ainsi qu'un branché, tout absorbé auditivement par son interlocuteur physiquement absent, peut visuellement en venir à fixer d'un regard absent une personne physiquement présente. Ce détachement peut 
alors être vécu par cette personne comme une forme de déni et a donc toute chance d'entraîner de sa part une réaction négative. Il est difficile d'aller plus loin dans cette hypothèse de couplage sensitif physique. Mais on peut aussi faire l'hypothèse d'un pur couplage psychologique et se demander si le regard du branché ne devient pas en partie insensible à son environnement afin de s'adapter au caractère invisible de son interlocuteur. Puisque celui-ci est invisible, ou seulement imaginable, le regard devient aveugle, ou introverti à fin de représentation. Quelle que soit l'explication, les conséquences sont les mêmes : une inattention à l'environnement ${ }^{15}$.

L'attitude liée à l'écoute emprunte un parcours différent, mais parvient au même but non recherché : la négation ou. tout au moins, l'effacement des présents au profit des absents. Pour entendre son interlocuteur, le branché a en effet tendance à vouloir s'isoler de ce qui devient pour lui le «bruit » ambiant. Il met une main sur son oreille libre, se tourne vers un mur. se penche en rentrant ses épaules, bref, fait dos à ce qui, normalement, devrait faire pour lui l'objet d'une attention certes distante mais réelle. Ou plus exactement, il y a bien attention, c'est-à-dire prise en compte des autres, mais elle consiste à adopter une attitude qui, en gros, veut dire : « vous me gênez ».

\section{Le déni}

C'est au demeurant bien la même impression que retirent les témoins de l'envol du branché. Pour eux, il y a bien décollement et éloignement du branché, et ce qu'il vit médiatiquement a toujours l'air d'être beaucoup plus important que sa relation avec son environnement physique. C'est en ce sens que le comportement du branché dans un lieu public peut paraître doublement "déplacé». D'une part, parce qu'il y a déplacement de son attention vers un point inaccessible aux co-présents et, d'autre part, parce qu'il rompt ainsi leur "être-ensemble", les règles tacites qui étaient associées au lieu ${ }^{16}$.

«La personne qu'on a en face et qui sort son téléphone mobile, on est complètement exclu de son monde. Moi, je me sens vraiment exclue quand quelqu'un, qui est dans le même espace que moi, parle avec un téléphone mobile. » «Dans un espace public, à mon avis, ce qui s'y passe doit être visible. Avec le mobile, on sort de cette visibilité, et je trouve que la règle du jeu n 'est pas respectée. Voilà ce qui me déplaît.»

«Les personnes qui partagent la même salle de restaurant ou de café, je ne les connais pas, je ne les connaîtrai probablement jamais. Mais il y a tout de même, me semble-t-il, quelque chose de commun : on est dans la même situation, on est tous à la même enseigne, on partage un même espace. À partir du moment où une de ces personnes est en communication téléphonique avec quelqu'un d'autre, elle sort d'une certaine manière de cet espace. Et je trouve que ça perturbe. » Le branché, par sa prise de distance ostentatoire, déchire le fragile tissu de sociabilité qui unissait les physiquement présents malgré leur silence et qui habillait leur sentiment d'exister ensemble dans leurs différences. C'est toute une forme de civilité qui menace alors de tomber en lambeaux. L'ailleurs étant inaccessible de façon conjointe, l'ici le devient aussi : il perd son statut d'évidence commune, de cadre aux interactions de co-présence productrices de son caractère public.

«Moi, ce qui me gêne, ce n'est pas le bruit que font ceux qui téléphonent. Ils ne font finalement pas plus de bruit que s'ils discutaient avec une personne qui serait en face d'eux. Ce qui me gêne, c'est de voir quelqu'un qui parle à quelqu'un d'autre, qui est ailleurs et que je ne vois pas. » «L'appel en public, c'est une forme de violence. Ça veut dire : j'ai plus important à faire.»

« En appelant comme ça, [le branché] me nie. J'ai l'impression d'être nié. » 
11 C'est dans le vécu de cette perte de l'éprouvé conjointement, dans cette rupture de sociabilité ou. tout au moins, dans sa déstabilisation et relativisation, que ce qui était jusqu'alors vécu comme implicite devient objet d'interrogations et d'ajustements. En ce sens, le mobile peut être considéré comme un bon révélateur des formes de l'êtreensemble propre aux lieux publics. Celui qui fréquente un lieu public s'attend en effet à $\mathrm{y}$ trouver une certaine ambiance, s'attend à ce que les présents se comportent d'une certaine façon dans la prise en compte de sa présence, de même qu'il s'attend aussi à ce que ceux-ci s'attendent à ce qu'il se comporte d'une certaine façon envers eux ${ }^{17}$.

Rompre cette attente réciproque revient à explicitement questionner le code qui, jusqu'alors, était «agi » de façon implicite. L'action déstabilisatrice révèle donc aux yeux de tous (comme un précipité peut révéler la présence d'un corps chimique) l'existence d'un code de conduite. Deux possibilités s'offrent alors aux présents. Soit ils acceptent que ce code soit transgressé, et les seules réactions observables sont adaptatives, soit ils refusent cette violation, et des réactions qu'elle suscite visent à explicitement rétablir le code implicite.

\section{Les réactions}

13 Nous avons pu observer que plus les réactions aux «envols » des branchés dans les lieux publics sont spontanées et démonstratives, et plus elles sont négatives. Elles visent toujours à dénoncer l'empiétement médiatique individuel sur l'espace physique commun. Ces réactions ont souvent lieu sous la forme d'une intervention orale, de la plus cinglante ( «Je suis dans un train ou à la poste?». "Vous voulez que je prenne des notes?») à la plus détachée ("Ça coûte cher la minute? ", «J'étais venu prendre un café... ») en passant par de l'humour («Vous lui direz bonjour de ma part »). par de la provocation («Autrefois, il y avait des panneaux: ne pas cracher par terre; je me demande s'il ne faudrait pas les restaurer : ne pas téléphoner à moins de dix mètres ») et même par du dédain ("Moi, je ne supporterais pas d'être sonné comme ça »). Mais ces réactions se donnent tout autant - si ce n'est plus - à voir par une communication non-verbale, une attitude corporelle critique ${ }^{18}$.

14 À cette fin, les jeux de regards sont particulièrement employés. Ainsi, il est très fréquent d'observer le passage d'un regard non focalisé (règle envers des inconnus dans un lieu public) à un regard focalisé si ce n'est insistant (d'habitude uniquement employé avec des personnes connues). Il y a, de la part du "plaignant», rupture volontaire de la distance attentionnée " généralement admise » dans un lieu public afin que le branché, par effet miroir, se rende compte qu'il est lui-même, par son attitude, en train de transgresser cette règle. Nous avons nous-mêmes pu éprouver, en jouant le rôle d'un branché sans gêne dans des lieux publics, combien ce type de regard insistant pouvait être ressenti comme une intrusion impolie de la part du plaignant. Pourtant, attirer l'attention d'un branché en pleine télécommunication ne va pas de soi! Rappeler publiquement à quelqu'un qu'il transgresse une règle n'est, en effet, déjà pas facile habituellement. Mais, avec un branché, il faut en plus transgresser la règle de distance attentionnée pour la faire respecter, ce qui n'est pas loin d'être paradoxal... Ceci explique sans doute que, dans bien ces cas, la gêne ressentie personnellement ne se concrétise pas par une plainte publique. 
15 Toujours au niveau des regards, il est une forme indirecte d'intervention d'une grande efficacité : le regard approbateur d'un ou de plusieurs co-présents vers le plaignant. Outre qu'il renforce la plainte par addition, ce regard tiers rappelle que la plainte n'est pas le fruit d'une susceptibilité individuelle, mais bien collective et qu'elle se réfère à quelque chose de socialement partagé. La plainte peut encore s'exprimer par un vacillement de l'expression, un froncement de sourcils, une moue de dépit, un soupir sonore, un geste d'exaspération ou une attitude de repli offensé. À chaque fois, il s'agit d'attirer l'attention du branché et de lui faire part de sa désapprobation, c'est-à-dire, en définitive, à le menacer de perdre la face s'il ne rectifie pas son attitude.

\section{Le rachat}

16 Il est des cas où la plainte peut ne pas être acceptée. Certains branchés prennent en effet d'entrée une attitude défensive visant à publiquement signaler qu'ils sont bien conscients qu'ils gênent, mais qu'ils ne peuvent pas faire autrement. En quelque sorte, ils font passer le fait qu'ils se savent «fautifs " pour une dispense d'action réparatrice. Il s'agit là d'un coup de force que seule une action d'une violence symbolique équivalente pourrait déstabiliser. Mais rares sont les plaignants potentiels disposés à risquer autant pour ce qui peut sembler si peu. Une attitude inverse peut mener au même résultat d'absence de réparation: au lieu de s'auto-désigner comme "fautifs innocents ", certains branchés mettent en représentation leur "totale absence ». Par une attitude à la limite de l'exhibition, ils signalent aux yeux de tous, qu'entièrement absorbés par l'ailleurs avec lequel ils communiquent, ils demeurent hors d'atteinte de quelque plainte que ce soit émanant de l'espace physique partagé. Regard absent, oreille libre bouchée et attitude de repli, fonctionnent comme autant de carapaces ne laissant aucune prise possible aux plaintes éventuelles.

17 Mais, dans la plupart des cas. les plaintes sont perçues et produisent leur effet. Le branché cherche alors à communiquer aux présents qu'il est « toujours là » malgré son absence flagrante, qu'il fait attention à eux, qu'il a éventuellement bien remarqué la réaction négative de certains à son égard, qu'il a bien conscience de l'incongruité de sa conduite et que celle-ci le plonge lui-même dans l'embarras. Sa réaction peut être immédiate: il abrège alors sa conversation et s'excuse ou bien quitte le lieu pour téléphoner. Mais la réaction est souvent différée. Se met alors en place un type d'échange - la plupart du temps gestuel - tout à fait inédit. Il s'agit pour le branché de faire comprendre à son entourage « qu'il va couper incessamment sous peu » et que, s'il ne le fait pas sur le champ, c'est non pas qu'il ne le veuille pas, mais parce que son interlocuteur « l'oblige » à poursuivre la conversation. En désignant le mobile, il peut souffler en se passant vivement le revers de la main libre sur la joue (« qu'est-ce qu'il est barbant ») ou faire un geste d'impuissance ou encore regarder fébrilement sa montre, comme si ce n'était pas lui mais l'urgence qui l'obligeait à ce regrettable écart d'urbanité. Ce type échange est à tendance schizophrénique car le dédoublement du branché est alors plus vécu comme une tension posant problème que comme un ersatz d'ubiquité libératrice. L'ailleurs ne s'accorde pas avec l'ici, alors que l'intérêt du mobile est précisément de pouvoir jongler avec les deux...

Lors d'interactions entre personnes se connaissant déjà bien (par exemple autour d'une table de café partagée avec des amis), l'irruption de l'ailleurs médiatique peut être perçue comme étant encore plus déplacée. Le branché signifie en effet clairement à ses 
vis-à-vis que l'urgence de l'appel l'emporte sur l'importance de leur conversation et que l'advenant téléphonique a priorité sur l'étant proxémique. Mais il est aussi plus facile pour lui de se justifier : en expliquant, par exemple, qu'étant de garde, il était impossible de ne pas répondre. Plus facile aussi de s'excuser: en prétextant par exemple avoir oublié d'éteindre son portable. Ou même de réparer l'impair à temps : en abrégeant la télécommunication avec une phrase destinée en fait aux co-présents (« excusez-moi, je ne suis pas seul») ou encore, si l'interlocuteur est connu des coprésents, en les faisant participer à la conversation.

\section{Conclusion : civilité télécommunicationnelle et lieux publics}

L'étude des réactions à l'usage des mobiles dans les lieux publics nous conduit à formuler deux remarques en guise de conclusion. La première a trait au devenir de telles réactions, la seconde à leur signification sociale. Le matériau sur lequel se base notre étude relève de pratiques émergeantes : les nouveaux utilisateurs de mobile n'ont cessé de se multiplier ces dernières années. Nous avons par ailleurs montré que, passée « l'euphorie » des premiers mois, la phase « égoïste » du branché (au court de laquelle l'individualité médiatique l'emporte sur la civilité proxémique) tend à s'estomper. Rapidement, une prise de conscience de la gêne occasionnée apparaît et un ajustement des conduites en fonction de la présence d'autrui est observable. Mais cet ajustement pèse à son tour peu face aux conduites ostentatoires des nouveaux utilisateurs... La construction progressive d'usages et en particulier de règles d'utilisation en public est sans cesse recouverte par la conduite "égoïste» des nouveaux utilisateurs, de telle sorte qu'un sans gêne de la téléphonie mobile paraît s'installer partout. Toutefois, le marché n'est pas indéfiniment extensible et l'on prévoit que, d'ici trois à cinq ans, il sera saturé. Les pratiques ne seront alors plus émergeantes, et il y a fort à parier que les conduites actuellement observables chez les " anciens utilisateurs» (qui se comptent tout de mène en millions) auront tendance à s'étendre à l'ensemble de la population des branchés ${ }^{19}$.

Or que nous apprennent ces conduites en termes de sociabilité dans les lieux publics? Essentiellement qu'un facteur discriminant semble présider le rapport entre réactions et lieux fréquentés ${ }^{20}$. Il est des lieux (par exemple certains cafés, les couloirs ou fumoirs des salles de spectacle, les galeries ou musées) où l'usage des téléphones mobiles, bien qu'officiellement toléré, est pratiquement désapprouvé, tandis que d'autres (par exemple les quais de gare, les salles d'embarquement, les rues passantes) où le même usage ne suscite aucune opposition. Toutes choses égales par ailleurs (et en particulier les dispositions proxémiques), plus les réactions sont nombreuses et négatives, et plus la réputation du lieu renvoie à une civilité sensible, à une attention partagée, à une atmosphère de sympathie sociale, à une ambiance positivement vécue, bref à un goût du lien social en public, autrement dit à un fort degré d'urbanité. Le mode «d'êtreensemble » dans ces lieux relève alors, au mieux d'une urbanité exquise et au pire d'une civilité appréciée. A l'inverse, moins les réactions sont nombreuses et négatives, et plus la réputation du lieu renvoie à sa simple disposition fonctionnelle, à une approche instrumentale de ses services, à une vision utilitariste de ses ressources, à une relation concurrentielle avec ceux qui le fréquentent, bref à la nécessité de les pratiquer selon 
une logique utilitariste. Le mode "d'être-ensemble» dans ces lieux relève alors, au mieux d'un ajustement bien compris, au pire d'une brutalité urbaine.

\section{BIBLIOGRAPHIE}

AUGOYARD J-F.. » Du lien social à entendre ». in Le lien social. Actes du XIII ${ }^{e}$ colloque de l'AISLF.

Genève, 1989, pp. 702-717.

GOFFMAN E., La mise en scène de la vie quotidienne, Paris. Minuit, 1973.

GOFFMAN E., Les rites d'interaction, Paris, Minuit. 1974.

HALL Ed. T., La dimension cachée. Paris, Seuil, 1971.

HALL Ed. T., Le langage silencieux, Paris. Mame, 1973.

JAUREGUIBERRY F., « Lieux publics, téléphone mobile et civilité », Réseaux, n 90. 1998, Paris, Hermes Sciences Publications.

JAUREGUIBERRY F., " Mobile telecommunications and the management of time ", Social Science Information, vol. 39. $\mathrm{n}^{\circ}$ 2, 2000. Londres. Sage Publications.

MEAD G. H., L Esprit, le Soi et la Société, Paris. PUF, 1963.

Ouere L. et Brezger D., « L'étrangeté mutuelle des passants ». Les Annales de la Recherche Urbaine, n 57/58, 1993, pp. 89-100.

SAPIR Ed., Anthropologie, Paris, Minuit, 1967.

SENNETT R., Les tyrannies de l'intimité, Paris, Seuil, 1979.

SCHÜTZ A., Le chercheur et le quotidien, Paris. Méridiens-Klincksieck, 1987.

\section{NOTES}

1. Les "téléphones mobiles" seront ici simplement appelés "mobiles", de même que les "utilisateurs du téléphone mobile» seront, pour plus de facilité, désignés par le tenue de «branchés ».

2. Les exceptions vont dans les deux sens sans qu'il soit possible de les quantifier: certains parlent certes plus fort, mais d'autres réduisent au contraire le volume de leur voix.

3. On peut estimer que la fin du caractère distinctif du mobile a, en gros, accompagné le dépassement du million d'utilisateurs (septembre 1995) et que sa banalisation commence avec le très net redressement de la courbe des abonnements à partir de l'automne 1997 (4 millions en septembre. 9 millions un an après. 16 millions deux ans après...).

4. Pour rendre compte de leur nouvelle expérience, les branchés ont souvent recours à une métaphore aérienne. Les appels nomades sont décrits comme autant $d$ " envols». de «décollages" délivrant de la pesanteur d'être lié à un lieu pour pouvoir communiquer. Les branchés se sentent "légers". comme détaches d'une contrainte physique qui devient alors relative. 
5. La méthode employée pour l'étude servant de base à l'exposition de ce qui suit a surtout été celle de l'observation passive. A cinq reprises, l'observation a toutefois été active : un sociologue se faisait passer pour un branché alors qu'un second filmait, au caméscope et de façon discrète, les réactions des personnes présentes. Ces scènes ont ensuite été étudiées image par image. Cette étude faisait partie d'une recherche beaucoup plus vaste qui portait sur l'expérience d'ubiquité télécommunicationnelle et qui, pour l'essentiel, reposait sur deux autres méthodes : l'entretien approfondi et l'intervention sociologique. Les informations recueillies par le biais de ces deux dernières méthodes ont en général recoupé celles que fournissait l'observation. A une exception (de taille...) près: les premiers branchés minimisaient totalement la gêne qu'ils pouvaient provoquer. Ce n'est qu'en fin de recherche (analyse réflexive, mais surtout généralisation des mobiles) qu'ils en prendront conscience. L'ensemble de cette recherche a été financée par le CNET et le CNRS, et menée à Paris, Pau, Strasbourg et Toulouse avec la collaboration de Nicole Lompré, François Ménard, Liliane Sochaki et Sandy Torres au sein du SET (Société Environnement Territoire, unité mixte de recherche 5603 du CNRS - Université de Pau).

6. Sennett R., 1979, p. 202.

7. Quéré L. et Brezger D., 1993, p. 91.

8. Quéré L. et Brezger D., 1993, p. 94.

9. Sur ce point, voir Jean-François Augoyard, 1989.

10. Goffman définit la «face» comme «la valeur sociale positive qu'une personne revendique effectivement à travers la ligne d'action que les autres supposent qu'elle a adoptée au cours d'un contact particulier» (Goffman, 1974, p. 9). C'est dire si maintenir la face est important pour toute individu : il en va rien moins que de son image sociale.

11. Goffman E., 1974, p. 8.

12. Sapir F... 1967 (première édition 1949), p. 46. Reprenant l'image. Edward T. Hall, 1973 (première édition 1959) parlera de « langage silencieux ».

13. Erving Goffman (1973), tome 2, p. 152.

14. C'est parce qu'ego pense qu'aller ferait idéalement la même expérience que lui de leur environnement commun s'il était à sa place (et réciproquement) que cet environnement lui (leur) apparait comme quelque chose de non seulement commun mais aussi partagé (voir Alfred Schütz, 1987).

15. Preuve indirecte: une récente étude de la protection routière montre que le temps de réaction d'un branché au volant est deux fois plus important que la normale...

16. Les citations d'usagers apparaissant dans ce texte sont toutes issues d'interviews menées clans le cadre de la recherche citée en note 5.

17. Cette "façon ", c'est-à-dire le soubassement social de cette attente réciproque, renvoi assez bien à ce que Georges Herbert Mead (1963) appelle «l'autrui généralisé ». Chacun agi ! certes de son propre concert, mais, ce faisant, en appelle a « l'autrui généralisé » qu'il a intériorisé au point où, la plupart du temps, celui-ci demeure inconscient.

18. Goffman appelle ce type de réactions des «actions d'amorçage». Elles visent à offrir à l'impoli « une dernière chance de remettre les choses en ordre et de faire tout de suite ce qu'il aurait dû faire avant de prendre des libertés » (Goffman, 1973, tome 2, p. 152).

19. Nous n'aurions évidemment pas écrit cela il y a cinq ans, lorsque les premiers utilisateurs étaient trop typés et pas assez représentatifs de la population générale.

20. Pour un développement de ce thème, voir Jauréguiberry F., 1998. 


\section{AUTEUR}

FRANCIS JAURÉGUIBERRY

SET/CNRS/Université de Pau 